\title{
Información para la equidad en salud en Chile ${ }^{1}$
}

\author{
Óscar Arteaga, ${ }^{2}$ Susan Thollaug, ${ }^{3}$ Ana Cristina Nogueira ${ }^{4}$ \\ y Christian Darras ${ }^{4}$
}

RESUMEN Objetivos. Estimar la magnitud de las desigualdades geográficas de salud en Chile mediante indicadores clave basados en datos e información de fácil obtención recolectada de forma rutinaria, y caracterizar la situación actual con respecto a la disponibilidad, calidad y accesibilidad de la información sobre equidad en salud recolectada de forma rutinaria por fuentes oficiales.

Métodos. Se usó un marco conceptual propuesto por la Organización Mundial de la Salud para el estudio de la equidad en salud que consta de cuatro dimensiones: I. estado de salud, II. determinantes de la salud, III. recursos y oferta del sistema de salud, y IV. utilización de servicios del sistema de salud. Para cada una de estas dimensiones se hizo una selección de indicadores para los cuales existía información disponible. La información, agregada por unidad geográfica (comuna, Servicio de Salud o región), fue analizada usando los siguientes métodos: análisis univariado (características de las distribuciones); análisis bivariado (correlaciones y tablas de frecuencia); tabulación de valores comunales para indicadores seleccionados.

Resultados. Estado de salud: encontramos una relación inversa entre la mortalidad y el ingreso familiar medio en la comuna $(\mathrm{r}=-0,24 ; \mathrm{P}<0,001 ; \mathrm{n}=191$ comunas). Determinantes de la salud: hay importantes variaciones entre las comunas con respecto al ingreso doméstico medio, años de escolarización, analfabetismo, calidad de la vivienda, abastecimiento de agua potable y sistemas de eliminación de aguas residuales. Recursos y oferta del sistema de salud: los gobiernos municipales de las comunas con mayores ingresos domésticos medios tienden a aportar mayores recursos financieros por beneficiario $(\mathrm{r}=0,19 ; \mathrm{P}=0,0130)$. El aporte del gobierno central, aunque se encuentra bien orientado, solo compensa parcialmente esta situación en beneficio de las comunas pobres. Utilización de servicios de atención de salud: entre los servicios de salud, el uso de los servicios de atención médica en el nivel primario es 2,8 veces mayor en unos que en otros, la atención médica de urgencia 3,9 veces mayor, y los egresos hospitalarios 2 veces más numerosos.

Conclusiones. Existen importantes variaciones geográficas en lo que se refiere a la mortalidad y a otros resultados de salud, en ingresos y condiciones ambientales, y en el financiamiento y utilización de los servicios de asistencia sanitaria. La información recolectada regularmente y que se encuentra disponible para caracterizar las variables relacionadas con la salud presenta con cierta frecuencia limitaciones de calidad, sostenibilidad o accesibilidad. En el contexto chileno, sería infructuoso centrar los mayores esfuerzos en la reorganización de los sistemas de información, toda vez que las pruebas ya existentes de marcadas desigualdades son suficientes para apoyar la planificación de intervenciones orientadas a mejorar con urgencia la situación de los chilenos más desprotegidos.

Palabras clave Equidad, Chile, información, estado de salud, determinantes de salud, recursos y oferta del sistema de salud, utilización de servicios del sistema de salud.

1 Trabajo desarrollado por la Representación de la Organización Panamericana de la Salud/Organización Mundial de la Salud (OPS/OMS) en Chile.

2 Escuela de Salud Pública de la Universidad de Chile, Santiago, Chile.

3 Consultora, OPS/OMS, Chile.

4 Representación OPS/OMS, Santiago, Chile.
La equidad ha sido uno de los principales sustentos filosóficos y valores que ha guiado el trabajo de la Organización Panamericana de la Salud desde su creación (1). Sin embargo, solo en años recientes ha aumentado en los foros académicos y políticos la preocupación por las inequidades en salud y sus factores determinantes, tanto entre los países como en cada 
uno de ellos $(2,3)$. Al mismo tiempo que se afirma que la equidad es un valor social fundamental, esta preocupación por el tema se caracteriza por estar menos basada en ideologías y más en datos objetivos orientados a reducir las pronunciadas diferencias en el estado de salud que existen hoy. Estas disparidades cobran aún más importancia después de varias décadas de un crecimiento del conocimiento médico sin paralelo en la historia de la medicina (4).

En Chile, el actual gobierno ha ubicado la equidad en el centro de las políticas que busca impulsar. Siendo la salud una de sus prioridades, el gobierno se ha comprometido con la reforma del sector como una de las siete grandes transformaciones que se propone realizar en el periodo 2000-2006 (5). La equidad constituye el eje en torno al cual se estructuran las propuestas específicas que considera la reforma (6).

Chile es un país de desarrollo intermedio que se encuentra en transición epidemiológica. Tiene una población de aproximadamente 15,2 millones de habitantes, de los cuales un $40 \%$ vive en la Región Metropolitana de Santiago, y un $85 \%$ en áreas urbanas. La población de las diferentes comunas es muy variable, desde poco más de un centenar de personas hasta más de 400000 habitantes. El producto interno bruto per cápita alcanzó 4603 dólares estadounidenses (USD) en el año 2000. Desde el punto de vista político-administrativo, el país se divide en 13 regiones, 51 provincias y 342 comunas, con un total de 178 ciudades de más de 5000 habitantes, 215 pueblos de 2000 a 5000 habitantes y 768 aldeas de menos de 2000 habitantes (7). El gobierno comunal está representado por la municipalidad. La organización de salud consta de 28 distritos sanitarios, denominados Servicios de Salud, que administran las políticas y programas de salud que define el Ministerio de Salud. Los límites de los Servicios de Salud no coinciden en todos los casos con los de otras divisiones administrativas.

En el presente artículo se presentan los resultados de un estudio realizado durante el año 2000 por un equipo téc- nico coordinado desde la Representación de la Organización Panamericana de la Salud/Organización Mundial de la Salud (OPS/OMS) en Chile. El estudio tuvo dos grandes objetivos: estimar la magnitud de las desigualdades geográficas en salud mediante indicadores clave basados en datos e información de fácil obtención recolectada de forma rutinaria, y caracterizar la situación actual con respecto a la disponibilidad de información, considerando aspectos de calidad (precisión, confiabilidad, oportunidad) y accesibilidad práctica, tanto para los investigadores como para el público en general. La hipótesis subyacente fue que el abordaje de los problemas de equidad supone un fortalecimiento de la capacidad de cada ciudadano de disponer de información significativa, pues sin el acceso a la información por parte de los ciudadanos, el tema queda como propiedad de los iniciados y se reducen las posibilidades de cambio.

\section{MATERIALES Y MÉTODOS}

El concepto de equidad en salud tiene una enorme amplitud y riqueza (8). La discusión se puede abordar desde varias dimensiones (9). El concepto de equidad en salud está particularmente enraizado en el conjunto de ideas y visiones de orden filosófico, ético, político y práctico que caracterizan la discusión contemporánea sobre equidad social. La equidad social se concibe como un factor que incrementa la estabilidad social y la productividad económica. Por el contrario, las inequidades en una sociedad y entre diferentes sociedades favorecen la tensión y el conflicto, todo lo cual resta recursos a esfuerzos constructivos y organizados (10). Usando este enfoque, la idea de equidad, que en ocasiones parece nebulosa, puede transformarse en una guía más pragmática para diseñar e implementar políticas.

La definición de equidad en salud adoptada en este estudio fue, por su simplicidad, la propuesta por Margaret Whitehead en 1990, que caracteriza a las inequidades como "diferencias que son innecesarias y evitables, y ade- más, consideradas injustas", enfatiza que la equidad es una noción normativa y señala la importancia de la no neutralidad en las elecciones y acciones (11). En otras palabras, el ideal de equidad obliga a la sociedad a sesgar su proceso de toma de decisiones para poder favorecer la equidad sobre la inequidad. Las prioridades de intervención son determinadas a través de procesos políticos en los que, inevitablemente, se reflejará su transparencia, la calidad de la información y la participación democrática $(12,13)$. Por considerar que complementa la definicón de Whitehead, también hemos usado la definición propuesta por la OPS desde la perspectiva de la organización de los servicios de salud, según la cual, equidad implica: "a) en una situación de salud, reducir al mínimo posible las diferencias evitables e injustas; b) recibir atención en relación con las necesidades (equidad de cobertura, acceso y uso) y contribuir de acuerdo a las capacidades de pago (equidad financiera)" (14).

Debido a su complejidad conceptual, la equidad en salud es un campo muy intrincado para la investigación empírica. Las complicaciones asociadas a la estimación de las inequidades provienen de diferentes ámbitos: multiplicidad de maneras de medir la salud; variedad de factores que afectan a la salud; diferentes tipos de subgrupos poblacionales a través de los cuales se pueden comparar los diferentes parámetros relacionados con la salud, y variados puntos de vista para evaluar la salud. Además, muchos de los parámetros mencionados pueden ser usados como variables dependientes o explicativas, dependiendo de las preguntas de investigación que se planteen. Más aún, muchas de estas variables están fuertemente relacionadas entre sí, con lo cual su análisis plantea grandes retos metodológicos (15-17).

Aunque está claro que existen diversas maneras de enfocar el análisis de la equidad para efectuar comparaciones, usando por ejemplo, variables de ingreso, género, étnicas (18) o combinaciones de estas variables, nuestra opción fue comparar diferentes unidades político-administrativas. Esta decisión 
se debió en parte a que dichas unidades constituyen en sí mismas espacios de construcción de políticas a través de las cuales se pueden desarrollar intervenciones. En Chile, la comuna es la unidad político-administrativa más básica y el gobierno municipal tiene autonomía con respecto a los gobiernos nacional y regional, en el contexto de un estado unitario y no federal. El nivel municipal es escenario de importantes decisiones respecto a la salud pública, pues los establecimientos de atención primaria están mayoritariamente entregados a la administración municipal.

Para poder alcanzar los objetivos de nuestro estudio, adoptamos el marco de análisis sugerido por la OMS (19) en 1998, que considera cuatro dimensiones: I. estado de salud, II. determinantes de la salud, III. recursos y oferta del sistema de salud, y IV. utilización de servicios del sistema de salud. Estas dimensiones fueron seleccionadas por un grupo de expertos reunido por la OMS, basándose en abundantes investigaciones que indican que las inequidades en las dimensiones II a IV pueden tener un importante impacto en la dimensión I. Elegimos este marco de análisis por varias razones: es sencillo, no corresponde a un modelo causal y no especifica vías de acción o interacciones. Aunque los modelos causales son cruciales para el diseño y dirección de intervenciones eficaces, no fue este el objetivo del presente estudio. Tam- poco desarrollamos ningún tipo de recolección primaria de información y nos centramos exclusivamente en las variaciones de algunos indicadores en Chile, sin comparar el estado de salud en Chile con el de otros países ni con indicadores internacionales.

\section{Datos y fuentes}

A partir de las cuatro dimensiones del marco de análisis, nos planteamos examinar una amplia gama de indicadores relacionados con la salud. La selección final de indicadores se hizo sobre la base de su disponibilidad a corto plazo. El cuadro 1 muestra los indicadores analizados, además de la co-

CUADRO 1. Marco de análisis del estudio: dimensiones de la equidad en salud, indicadores seleccionados, nivel de agregación y fuentes de los datos para cada dimensión

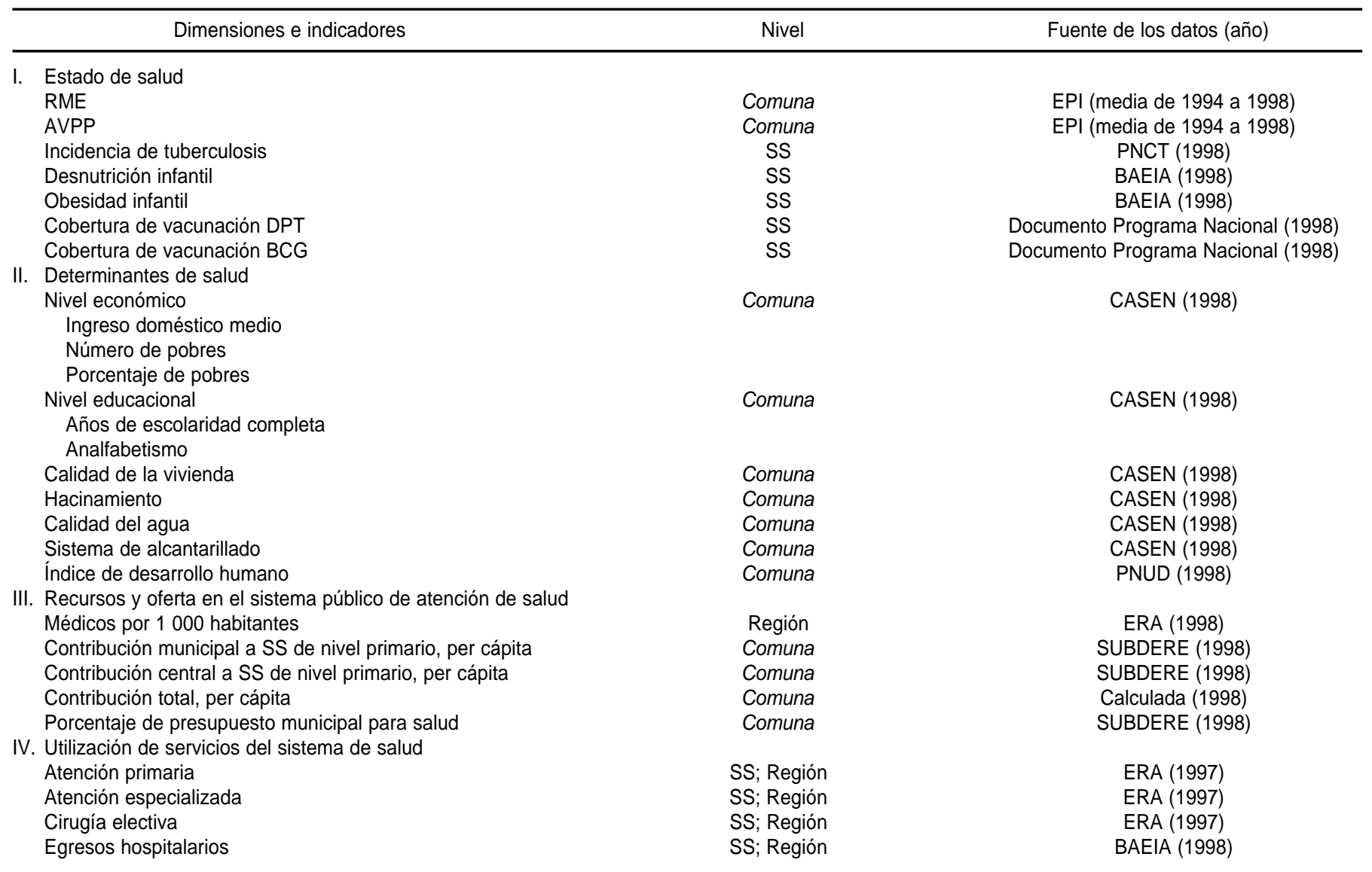

RME: razón de mortalidad estandarizada. EPI: Departamento de Epidemiología, Ministerio de Salud. AVPP: años de vida potencial perdidos. PNCT: Programa Nacional de Control de la Tuberculosis, Ministerio de Salud. SS: Servicio de Salud. BAEIA: Boletín Anual de Estadísticas e Indicadores de Atención, Ministerio de Salud. DTP: difteria, tétanos, tos ferina. BCG: bacilo de Calmette-Guérin. CASEN = Encuesta de Caracterización Socioeconómica Nacional, Ministerio de Planificación. PNUD: Programa de Naciones Unidas para el Desarrollo. ERA: Estudios de Red Asistencial, Ministerio de Salud. SUBDERE: Subsecretaría de Desarrollo Regional y Administrativo, Ministerio del Interior. 
rrespondiente fuente de datos y unidad geográfica de agregación. En la práctica, para muchos de los indicadores que inicialmente quisimos analizar (entre ellos varios sugeridos por la OMS en el marco metodológico usado en este trabajo) no encontramos fuentes de datos adecuadas. Los indicadores para los cuales se pudo encontrar información fueron agrupados según la unidad administrativa más pequeña para la cual existía información disponbible. Los niveles de agregación fueron: comuna, Servicio de Salud o región.

La información que usamos procede en la mayoría de los casos de fuentes oficiales (cuadro 1). La mayoría de los datos fueron obtenidos en formato electrónico, entre ellos los datos de mortalidad y utilización de servicios del sistema de salud, los datos financieros y los de la Encuesta de Caracterización Socioeconómica Nacional de Hogares (CASEN), 1998. Sin embargo, la información sobre varios indicadores debió extraerse de publicaciones impresas. Todos los datos que se presentan en este estudio representan cifras agregadas, calculadas por los organismos que aportaron la información. Con la excepción de las razones de mortalidad estandarizadas (RME), los datos proporcionados por las fuentes respectivas no se acompañaron de información sobre la variabilidad de los mismos.

Los valores de las RME usados en este trabajo fueron estimados por el Departamento de Epidemiología del Ministerio de Salud de Chile (20) y tienen una base poblacional. Los valores de las tasas de mortalidad comunal corresponden al promedio para el quinquenio 1994-1998 y la población de referencia que se usó para los cálculos de las RME corresponde a 1996. El indicador de mortalidad AVPP (años de vida potencial perdidos) está basado en una expectativa de vida de 80 años y fue estimado por el Departamento de Epidemiología del Ministerio de Salud a partir de la misma información de mortalidad comunal. Las estimaciones de la población de las comunas corresponden a las oficiales que proyecta el Instituto Nacional de Estadísticas a partir del último censo nacional de población (1992).
La encuesta CASEN, 1998, que viene siendo efectuada bianualmente desde 1987 por el Ministerio de Planificación, fue la principal fuente de la información socioeconómica analizada en este estudio. Algunos de los contenidos investigados en la encuesta han ido cambiando significativamente, lo que dificulta la realización de estudios longitudinales. Aunque en 1998 la encuesta fue representativa del $90 \%$ de la población chilena, solo incluyó 198 de las 342 comunas del país. Las comunas no representadas corresponen a las de población rural y dispersa. También se utilizaron como fuentes las publicaciones del Departamento de Estadísticas y de la División de Inversiones y Desarrollo de la Red Asistencial del Ministerio de Salud, y las bases de datos de la Subsecretaría de Desarrollo Regional y Administrativo (SUBDERE) del Ministerio del Interior.

\section{Métodos}

En este estudio, de tipo transversal, ecológico, se usan indicadores relacionados con la salud agregados al nivel de comuna, Servicio de Salud o región. En el análisis de la información se usaron los siguientes métodos: 1) análisis univariado (características de las distribuciones); 2) análisis bivariado (correlaciones y tablas de frecuencia); 3) tabulación de valores comunales para indicadores seleccionados, y 4) mapeo de variaciones comunales de las tasas de mortalidad. Los programas informáticos usados en el proyecto fueron: SAS, EpiMap, MSExcel, MSPowerPoint y MSWord.

Para la mayoría de los indicadores analizados se calcularon los valores mínimos, máximos y medianos, y la razón entre los valores mínimo y máximo. Los datos habían sido agregados previamente por las fuentes de información $\mathrm{y}$, en consecuencia, ninguno de los valores representa información individual, de hogares ni de personas. En la presentación de los resultados también se incluyen indicadores cuya información de respaldo era problemática. Estas cifras basadas en información incompleta o con problemas de confiabilidad se muestran en cursiva en los cuadros.

\section{RESULTADOS}

\section{Dimensión I: estado de salud}

Las estadísticas descriptivas de los indicadores de esta dimensión se resumen en el cuadro 2. Los valores extremos resultantes de cifras pequeñas $o$ datos incompletos fueron eliminados del análisis. Las tasas de mortalidad estandarizadas por edad y sexo presentan una gran variación entre comunas a nivel nacional y dentro de cada región, incluyendo la Región Metropolitana de Santiago. Después de eliminar los valores extremos, la variación de las RME comunales oscila entre 30 y 160 en relación con el promedio nacional estandarizado de 100. Encontramos una relación inversa entre la mortalidad (RME) y el ingreso doméstico medio de las comunas reflejado en la encuesta CASEN, 1998, $(r=-0,24$; $P<0,001 ; n=191$ comunas). Es importante destacar que la encuesta CASEN, 1998, no aporta información sobre seis de las siete comunas con la mortalidad más alta del país, pues coresponden a zonas no representadas en la encuesta. Esto constituye una señal de que no se estaría recogiendo información sobre algunas poblaciones más desfavorecidas. El análisis del indicador AVPP también mostró una enorme variación y una relación inversa con el ingreso doméstico medio (figura 1).

$\mathrm{El}$ indicador de enfermedades infecciosas analizado (incidencia de tuberculosis por 100000 habitantes) mostró diferencias de más de cinco veces entre los 28 Servicios de Salud. Sin embargo, estas cifras deben interpretarse con cautela, pues la incidencia nacional de tuberculosis en Chile es muy baja, debido a lo cual se relativiza el valor de las diferencias. Los datos acerca del estado nutricional de los niños atendidos en el sistema público en 1998 indican que el porcentaje de desnutrición infantil tiene variaciones de hasta cinco veces entre los Servicios de Salud y que el porcentaje de niños obesos oscila entre el $4,2 \%$ y el $10,9 \%$. No obstante, 
CUADRO 2. Resumen de la estadística descriptiva de la dimensión I (estado de salud). Chile, 1998a

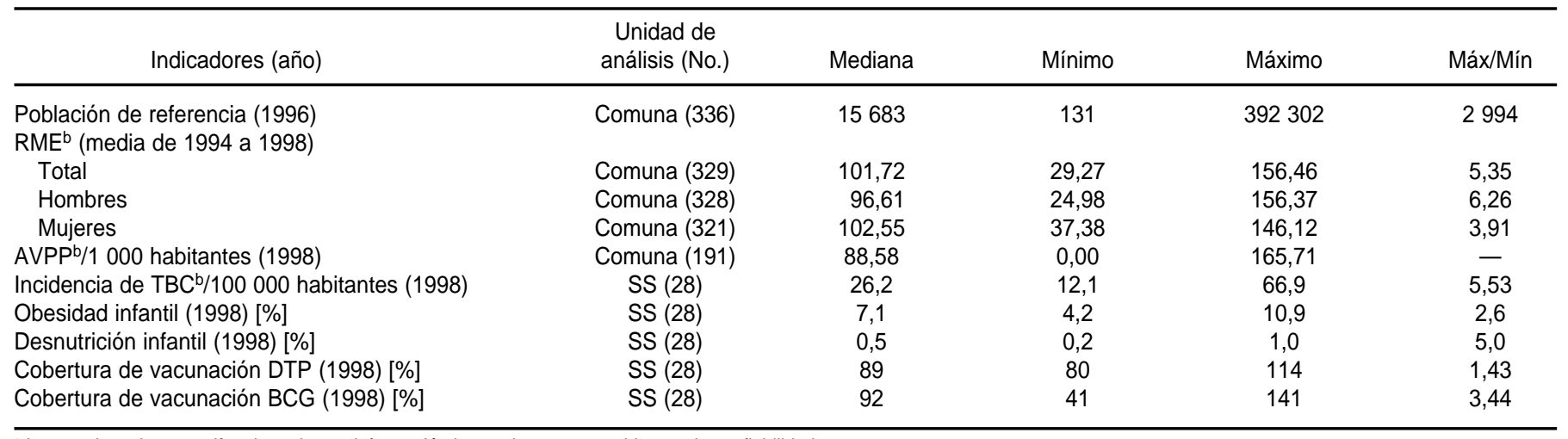

a Las cursivas denotan cifras basadas en información incompleta o con problemas de confiabilidad.

b RME: razón de mortalidad estandarizada. AVPP: años de vida potencial perdidos. TBC: tuberculosis. DTP: difteria, tétanos, tos ferina. BCG: bacilo de Calmette-Guérin.

FIGURA 1. Años de vida potencial perdidos (AVPP)/1000 habitantes e ingreso doméstico mensual medio: distribución conjunta de valores comunales, ordenados por AVPP. Chile, 1998

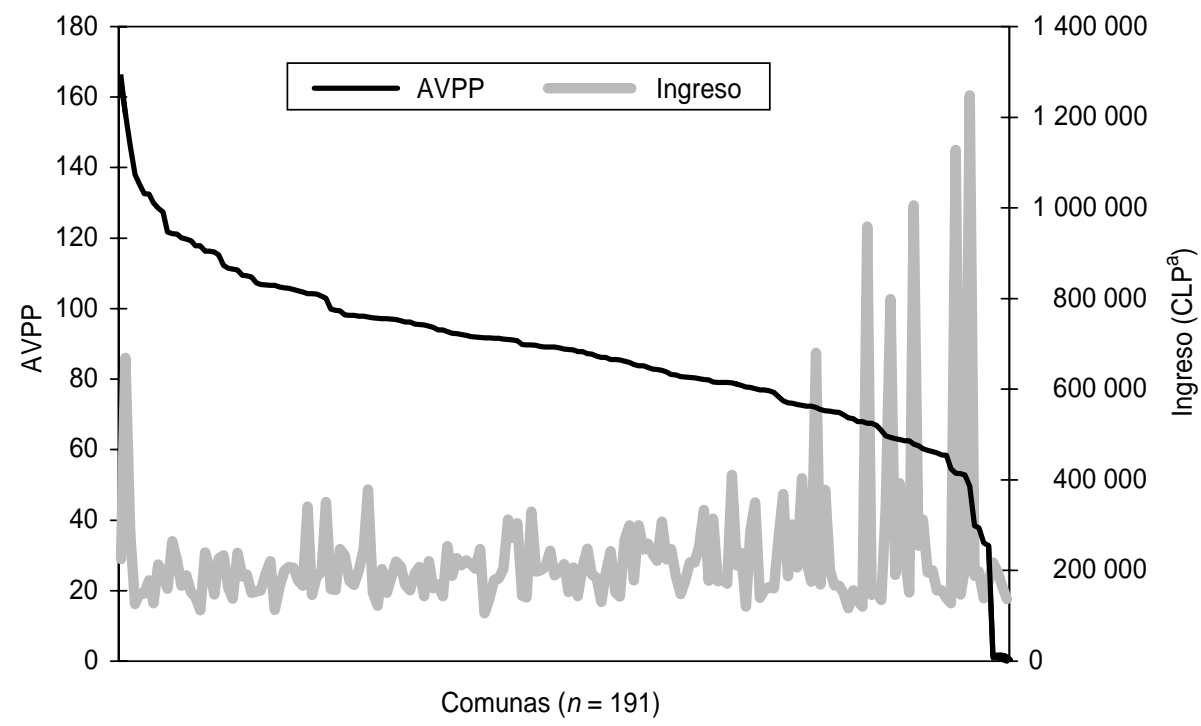

Fuentes: Departamento de Epidemiología, Ministerio de Salud y Encuesta de Caracterización Socioeconómica Nacional de Hogares (CAESEN), 1998.

a CLP: pesos chilenos.

existen dudas acerca de la precisión de estas cifras, así como de la información sobre las coberturas de inmunización, pues a pesar de que están basadas en publicaciones recientes, los denominadores usados corresponden a poblaciones estimadas. Las imprecisiones en las estimaciones probablemente expliquen que haya registros de coberturas máximas de vacunación con BCG (bacilo de Calmette-Guérin) y DPT (difteria, téta- nos, tos ferina) superiores al $100 \%$ en algunas unidades geográficas.

\section{Dimensión II: determinantes de la salud}

Todos los datos examinados en esta dimensión (cuadro 3) fueron tomados o están basados en la información de la encuesta CASEN, 1998. Estos datos están agregados a nivel comunal y su confiabilidad es alta. Es importante recordar que el análisis de estos datos excluye 151 comunas rurales (con el 10\% de la población de Chile) no representadas en la encuesta. A pesar de ello, encontramos importantes variaciones en todos los indicadores examinados.

La distribución de los ingresos, incluso cuando se representa como "ingreso doméstico medio de la comuna", tiene una enorme desviación a la derecha (figura 2). De acuerdo con los datos de la encuesta CASEN, 1998, solo un $6 \%$ de la población vive en comunas con un ingreso doméstico medio superior a 400000 pesos chilenos (CLP)/mes ( USD 800). Las únicas cuatro comunas con un ingreso doméstico mensual medio superior a CLP 1000000 se encuentran todas en el sector oriental de Santiago. A la vez, la mayor concentración de pobres también se observa en la Región Metropolitana. Al dividir la distribución de ingresos domésticos mensuales de las comunas en deciles, el límite superior del segundo decil es CLP 227 000/mes ( USD 400). De acuerdo con las cifras de la encuesta CASEN, en 1998 el 55\% la población de Chile vivía en comunas en las cuales el ingreso familiar medio era inferior a ese nivel. Además, es probable que la población de comunas no representadas en la encuesta (población rural y geográficamente dispersa) también corresponda a comunas de bajos ingresos. 
CUADRO 3. Resumen de la estadística descriptiva de la dimensión II (determinantes de salud). Chile, 1998

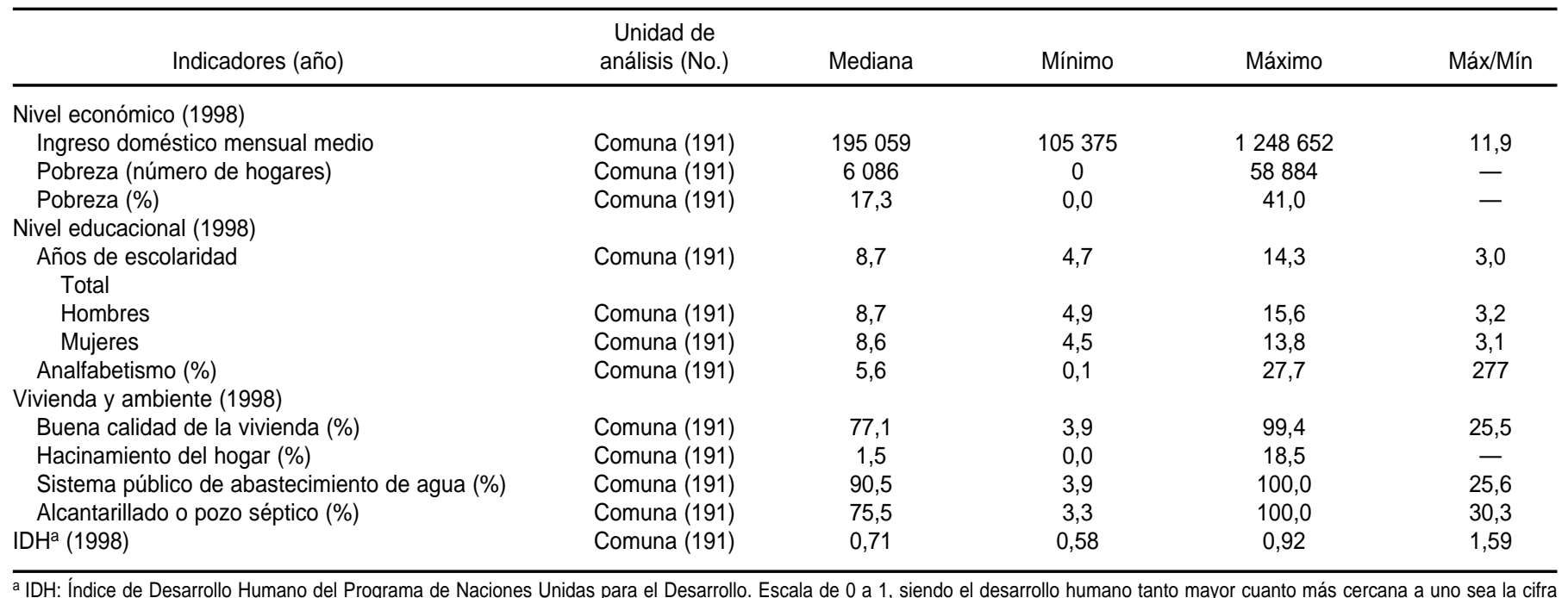
comunal.

El máximo valor de años de escolarización fue similar para los hombres y las mujeres en las comunas, pero al comparar las comunas entre sí, las cifras medias variaron alrededor de tres veces, desde 4,7 a 14,3 años. La variación del analfabetismo también fue enorme: $0,1 \%$ a $27,7 \%$.

En lo que se refiere a las condiciones físicas y ambientales básicas de la vivienda, los datos de la encuesta CASEN, 1998, muestran diferencias muy marcadas entre las comunas con respecto a la calidad de la vivienda, al abastecimiento de agua potable y a los sistemas de eliminación de aguas residuales (cuadro 3). Mientras que en algunas comunas prácticamente todos los hogares cumplían o superaban el estándar respectivo, en 20 comunas las viviendas de buena calidad representaban menos del 50\%.

\section{Dimensión III: recursos y oferta del sistema de salud}

Los cinco indicadores que se incluyen en esta sección (cuadro 4) solo representan un pequeño conjunto de medidas posibles para evaluar el funcionamiento de los sistemas de salud desde la perspectiva de la oferta de servicios. El único indicador de recur-

FIGURA 2. Ingreso doméstico mensual medio por comunas. Chile, 1998

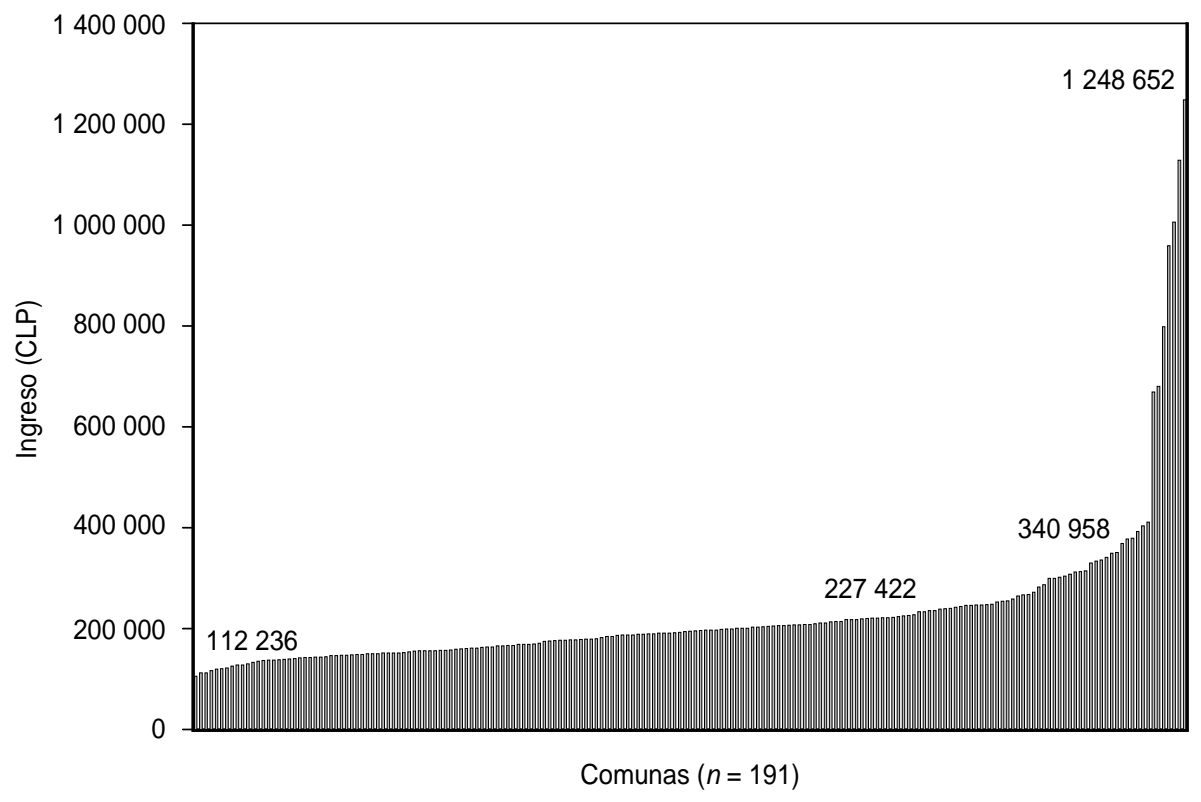

Fuente: Encuesta de Caracterización Socioeconómica Nacional de Hogares (CASEN), 1998. CLP: Pesos chilenos.

sos humanos que incluimos en esta dimensión fue la tasa de médicos, agregada a nivel de región, pues no fue posible obtener información completa por Servicio de Salud o comuna.

Entre las diferentes regiones de Chile existe una diferencia de casi siete veces en la tasa de médicos del sector público: desde 0,28 hasta 1,92 médicos por 1000 habitantes. Debe destacarse que la mayoría de los médicos de Chile se desempeñan tanto en el sector público como en el sector privado, haciendo difícil estimar el acceso real a 
CUADRO 4. Resumen de la estadística descriptiva de la dimensión III (recursos y oferta de servicios de salud). Chile, 1998 ${ }^{a}$

\begin{tabular}{|c|c|c|c|c|c|}
\hline Indicadores (año) & $\begin{array}{c}\text { Unidad de } \\
\text { análisis (No.) }\end{array}$ & Mediana & Mínimo & Máximo & Máx/Mín \\
\hline Médicos/1 000 habitantes (1998) & Región (13) & 0,92 & 0,28 & 1,92 & 6,9 \\
\hline \multicolumn{6}{|c|}{ Financiamiento del sistema público municipal de salud (1998) } \\
\hline Contribución ${ }^{b}$ del MINSALC per cápita (CLPd) & Comuna (262) & 10566 & 881 & 133177 & 151 \\
\hline Contribución ${ }^{b}$ municipal per cápita (CLP) & Comuna (302) & 4031 & 13 & 155201 & 11601 \\
\hline Contribución b combinada per cápita (CLP) & Comuna (304) & 13004 & 175 & 268926 & 1537 \\
\hline Presupuesto municipal destinado a salud (\%) & Comuna (300) & 5,0 & 0,0 & 24,0 & - \\
\hline
\end{tabular}

médicos que pueden tener los diferentes grupos de población en un área determinada.

El financiamiento de la atención primaria pública en Chile se hace mediante una combinación de fondos que provienen del Ministerio de Salud y de cada municipalidad. Solo el aporte central está normado por un marco legislativo, lo que hace que, en la práctica, el total sea determinado localmente. Como se explicó antes, la opción de este estudio fue trabajar con la información disponible en los sistemas regulares de recolección y manejo de la misma. En este sentido, la información proporcionada por la SUBDERE del Ministerio del Interior resultó ser bastante incompleta, con ausencia de datos de alrededor de un $10 \%$ para los aportes municipales a la atención primaria. Aún así, las diferencias en los fondos disponibles para las diferentes comunas fueron importantes. Expresando los datos per cápita por beneficiario público, estas variaciones se verificaron tanto para el aporte central como para el aporte municipal y el aporte combinado de ambas fuentes. Por otra parte, también se encontró una enorme variación en el porcentaje del presupuesto municipal total que las municipalidades destinan a la salud, siendo varias las municipalidades que destinan menos del $1 \%$ a los establecimientos de salud que administran. El análisis de correlación (cuadro 5) solo fue estadísticamente significativo para el aporte municipal $(r=0,19 ; P=$ 0,0130), es decir, la información analizada indica que las comunas con

CUADRO 5. Correlación entre ingreso doméstico medio comunal ${ }^{\mathrm{a}}$ y financiamiento de la atención primaria municipal

\begin{tabular}{lrcc}
\hline \multicolumn{1}{c}{ Variable } & $r$ & $P$ & Comunas (No.) \\
\hline Contribución del MINSAL & $-0,08$ & 0,3527 & 134 \\
Contribución municipal & 0,19 & 0,0130 & 172 \\
Contribución combinada & 0,07 & 0,3315 & 168 \\
Presupuesto municipal destinado a salud (\%) & $-0,04$ & 0,6385 & 170 \\
\hline
\end{tabular}

a Datos de la Encuesta de Caracterización Socioeconómica Nacional de Hogares (CASEN) 1998; $n=196$ comunas.

mayores ingresos domésticos medios tienden a aportar mayores recursos financieros por beneficiario. El aporte del gobierno central, aunque se encuentra bien orientado, en el mejor de los casos solo compensa parcialmente esta situación en beneficio de las comunas pobres. Dicho de otro modo, el esfuerzo que pueda hacer el gobierno central para corregir inequidades focalizando recursos en comunas pobres se ve atenuado por el mayor aporte que hacen las comunas de mayores recursos a sus establecimientos de salud.

\section{Dimensión IV: utilización de servicios del sistema de salud}

En esta dimensión (cuadro 6), encontramos una pronunciada variación de las tasas de uso en los niveles regional y de Servicios de Salud. Entre los 28 Servicios de Salud de Chile, el uso de los servicios de atención primaria varía 2,8 veces, la atención médica de urgencia 3,9 veces y los egresos hospitalarios 2 veces. La figura 3 ilustra la variación en el uso de atención médica de nivel primario entre los Servicios de Salud. Resulta particularmente interesante observar que los seis Servicios de Salud de la Región Metropolitana muestran considerables diferencias en el uso de atención médica de nivel primario.

Es importante destacar que los datos de utilización de servicios del sistema de salud que se presentan en este trabajo solo reflejan el uso en el sector público. Sin incorporar información sobre el sector privado no es posible conocer la variación total que existe entre las diferentes unidades geográficas del país. Sin embargo, incluso dentro del sistema público, las variaciones geográficas resultan muy importantes.

\section{DISCUSIÓN}

\section{Disponibilidad, calidad y pertinencia de la información}

Aunque los hallazgos de este y de otros estudios (21-23) muestran importantes desigualdades en el estado de salud y sus determinantes en Chile, la panorámica todavía resulta bastante 
CUADRO 6. Resumen de estadística descriptiva. Dimensión IV: utilizacion de servicios de atencion de salud

\begin{tabular}{|c|c|c|c|c|c|}
\hline Indicadores (año) & $\begin{array}{l}\text { Unidad de } \\
\text { análisis (No.) }\end{array}$ & Mediana & Mínimo & Máximo & Máx/Mín \\
\hline \multicolumn{6}{|l|}{ Cobertura de seguros, sistemas público y privado, 1998} \\
\hline Público \% & $\begin{array}{c}\text { Comuna (191) } \\
\text { Región (13) }\end{array}$ & $\begin{array}{c}70,8 \\
66\end{array}$ & $\begin{array}{c}15,2 \\
46\end{array}$ & $\begin{array}{c}95,8 \\
71\end{array}$ & $\begin{array}{l}6,30 \\
1,53\end{array}$ \\
\hline Isapre \% & $\begin{array}{c}\text { Comuna (191) } \\
\text { Región (13) }\end{array}$ & $\begin{array}{c}13,4 \\
17\end{array}$ & $\begin{array}{l}1,4 \\
12\end{array}$ & $\begin{array}{c}85,3 \\
42\end{array}$ & $\begin{array}{l}62,4 \\
3,65\end{array}$ \\
\hline Egresos hospitalarios/100 habitantes & $\begin{array}{l}\text { Región (13) } \\
\text { SS (28) }\end{array}$ & $\begin{array}{l}13,3 \\
12,6\end{array}$ & $\begin{array}{c}10,5 \\
9,1\end{array}$ & $\begin{array}{l}16,7 \\
18,2\end{array}$ & $\begin{array}{l}1,59 \\
2,00\end{array}$ \\
\hline Atención primaria: atenciones/1 000 beneficiarios & $\begin{array}{l}\text { Región (13) } \\
\text { SS (28) }\end{array}$ & $\begin{array}{l}1136 \\
1167\end{array}$ & $\begin{array}{l}817 \\
747\end{array}$ & $\begin{array}{l}2062 \\
2062\end{array}$ & $\begin{array}{l}2,5 \\
2,8\end{array}$ \\
\hline Atención especializada: atenciones/ 1000 beneficiarios & $\begin{array}{l}\text { Región (13) } \\
\text { SS (28) }\end{array}$ & $\begin{array}{l}503 \\
518\end{array}$ & $\begin{array}{l}340 \\
276\end{array}$ & $\begin{array}{c}746 \\
1474\end{array}$ & $\begin{array}{l}2.2 \\
5,3\end{array}$ \\
\hline Atención de urgencias: atenciones/ 1000 beneficiarios & $\begin{array}{l}\text { Región (13) } \\
\text { SS (28) }\end{array}$ & $\begin{array}{l}641 \\
656\end{array}$ & $\begin{array}{l}539 \\
375\end{array}$ & $\begin{array}{l}1017 \\
1473\end{array}$ & $\begin{array}{l}1,9 \\
3,9\end{array}$ \\
\hline
\end{tabular}

FIGURA 3. Tasas de utilización de atención médica de nivel primario por Servicio de Salud. Atenciones médicas por 1000 usuarios

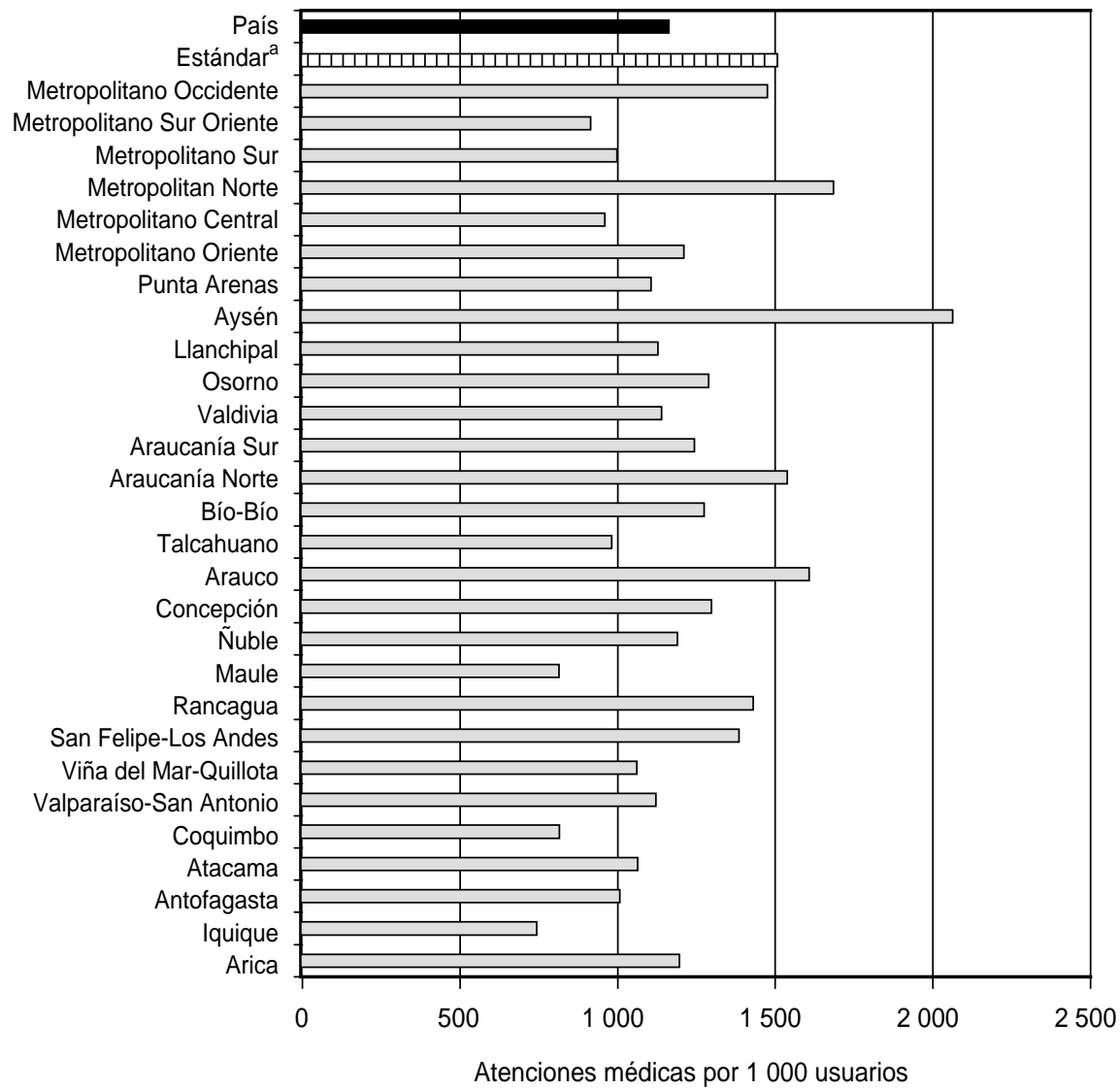

Fuente: División de Inversiones y Desarrollo de la Red Asistencial, Ministerio de Salud de Chile.

a Estándar es la norma establecida por el Ministerio de Salud de Chile para las atenciones médicas por persona y año en e nive primario de atención. Existe un estándar para los programas Infantil, del Adulto y de la Mujer. Para la población en genera corresponde a 1,5 atenciones médicas de nivel primario por persona y año. Para simplificar el análisis se multiplica por 1000. general. Aunque existe más información que la que pudo analizarse en este estudio, a partir de nuestra experiencia, nos parece que la actual información de base para llevar a cabo estudios sobre inequidades en salud resulta limitada en términos de su accesibilidad, suficiencia y, en algunas instancias, calidad.

Un obstáculo mayor para poder desarrollar estudios de mayor profundidad sobre la equidad en Chile procede de la falta de información a nivel de hogares, es decir, de información que permita combinar el análisis de factores demográficos, socioeconómicos y ambientales con información de buena calidad sobre las conductas de salud y la utilización de los servicios del sistema de salud a nivel de hogares o personas individuales. Los análisis ecológicos constituyen un instrumento intrínsecamente más débil para estudiar las relaciones entre variables que pueden existir en las poblaciones.

Al menos hasta el momento de realizar este estudio, la principal encuesta de hogares en Chile, CASEN, hacía solo una limitada contribución a las bases de información sobre aspectos importantes relacionados con la salud, pues no tiene un objetivo específico de salud y las pocas preguntas sobre este tema tienden a ser un tanto ambiguas 
y vagas. Los autores de un estudio reciente basado en los datos de la encuesta CASEN, 1998, destacan que, por ejemplo, el único ítem referido al uso de servicios de salud combina todos los tipos de contactos de atención de salud en los últimos 3 meses, no siendo posible diferenciar entre atención perinatal, control de salud de niños sanos, control de morbilidad o traumatismos (24). Por otro lado, en la encuesta se hacen varias preguntas destinadas a medir la satisfacción de los usuarios con los servicios públicos de atención de salud, pero la naturaleza de las preguntas hace que la información resultante sea difícil de interpretar, pues a los entrevistados se les pregunta si perciben mejorías en los servicios ofrecidos por el sistema público de salud en los últimos 4 años, sin que se especifiquen criterios para poder hacer dichas comparaciones.

Debido a que la transparencia de la presupuestación constituye un elemento clave para poder promover servicios públicos que sean equitativos, resulta vital que este tipo de información sea completa, precisa y fácilmente disponible para el público (25).

Mucha de la información que las agencias de gobierno ponen a disposición del público se publica en versiones impresas. En nuestro análisis encontramos una carencia de información sobre la variabilidad de las cifras resumen y estimaciones entregadas en las publicaciones. Además, las publicaciones impresas no incluían los denominadores -o referencias de ellos-, necesarios para poder hacer algunos análisis estadísticos básicos. De igual modo, sería muy útil disponer de más información sobre cómo se recolectaron y registraron los datos, incluidas las formas de muestreo cuando sea relevante, para poder interpretar los datos seleccionando las formas más adecuadas de analizarlos. Es frecuente que los registros de datos oficiales se encuentren agregados en unidades geográficas grandes, como la región, lo que puede obscurecer la variabilidad subyacente de las variables que se estudian.

En el transcurso del presente estudio pudimos constatar algunas dificul- tades en la obtención de la información en formato electrónico, que es la forma más útil para el análisis. Otras dificultades incluyeron la poca planificación previa de algunas agencias de gobierno para poner sus datos a disposición de los investigadores y el público en general, así como una cierta falta de procedimientos estandarizados o poco expeditos para poder acceder a la información.

Un hecho que resalta en cualquier estudio que requiera analizar información proveniente de diversos sectores de organización del Estado en Chile es que los límites de las unidades administrativas de los diferentes sectores no son coincidentes en las unidades territoriales inferiores al nivel regional. Esto constituye un obstáculo complicado a la hora de combinar información proveniente de los registros de un tipo de unidad (por ejemplo, los Servicios de Salud), con datos provenientes de otro sector (por ejemplo, la Dirección Provincial de Educación). Otro factor relacionado con el punto previo es que no siempre los registros oficiales cumplen con las normativas de estandarización de nombres en las bases de datos que se manejan en las diferentes reparticiones públicas. No es infrecuente que algunas comunas, especialmente aquellas con nombres compuestos, tengan varias alternativas en los registros, lo que dificulta, o al menos retrasa, el manejo y análisis de la información.

Las dificultades para poder comparar la información del sector público y privado de salud también representan una limitación mayor en el estudio de aspectos importantes de equidad en salud en Chile. Es posible que al comparar los sectores público y privado, que en su conjunto constituyen el sector de la salud chileno, existan aún mayores variaciones que las observadas en este estudio para el sector público. Aunque en definitiva ambos sectores están sujetos a políticas públicas que los afectan, es mucho más difícil obtener información acerca del sector privado que del público.

Cuando la información no es fácilmente asequible a los investigadores se pierden oportunidades de retroalimentación sobre la situación del sector de la salud. Esto puede ser contraproducente, pues investigadores independientes, grupos de interés público $\mathrm{y}$, en definitiva, todos los ciudadanos interesados en participar en el proceso político, podrían restar sus esfuerzos de apoyo a políticas que buscan promover la equidad.

En los últimos tiempos existen algunos signos iniciales que favorecen un mejor aprovechamiento de la información para apoyar y avanzar en políticas públicas a través de la investigación. En la actualidad se encuentran en pleno desarrollo varias iniciativas que apuntan a mejorar los sistemas de información en Chile. El Ministerio de Salud está revisando y consolidando sus sistemas de información. El Instituto Nacional de Estadísticas y el Ministerio de Salud desarrollaron y aplicaron una nueva encuesta de hogares centrada en la calidad de vida y la salud, cuyos resultados se conocerán próximamente. La SUBDERE del Ministerio del Interior está implementando un Sistema Nacional de Indicadores Municipales (SINIM), cuyo propósito es sistematizar un conjunto de indicadores que refleje el desempeño del municipio en áreas clave (salud, educación, desarrollo social, desarrollo territorial, administración y finanzas), aportando información útil para apoyar la gestión y la toma de decisiones de todos los actores involucrados con el municipio. Además, en el último año, varias agencias de gobierno han puesto a disposición del público información útil en sus sitios de Internet, lo cual expresa un cambio en la disposición de compartir la información con el conjunto de la ciudadanía.

\section{Hallazgos relevantes desde la perspectiva de la equidad}

En nuestro estudio, encontramos importantes variaciones geográficas en todos los indicadores de salud y relacionados con la salud que examinamos. El hallazgo más impactante, documentado también en otros trabajos, 
es la enorme desviación de la distribución de los ingresos en el país. Los ingresos domésticos medios de las comunas varían hasta 12 veces. Los AVPP presentaron una correlación inversa y significativa con el nivel socioeconómico. Más aún, cada uno de los indicadores educacionales y ambientales que examinamos, todos ellos conocidos factores determinantes de la salud, tuvieron una fuerte correlación con el ingreso doméstico medio comunal. Esto indicaría que, si bien existen algunas medidas compensatorias, incluso los beneficios del sistema público están todavía orientados hacia quienes se encuentran en mejor situación. Además, las grandes disparidades socioeconómicas parecen tener un impacto negativo en la salud, independientemente de los niveles de ingreso absoluto (26).

Encontramos importantes disparidades en el financiamiento de la salud en el país. Un estudio reciente de Larrañaga e Infante muestra que la descentralización del gobierno se ha asociado a resultados inesperados en términos de la distribución de los recursos del sistema público (27). Estos investigadores verificaron que, si bien la fórmula de asignación de recursos desde el nivel central a las municipalidades es progresiva y está definida por ley, hay otros factores, como el tamaño poblacional y la influencia política, que tienen un fuerte impacto sobre la distribución final de los recursos a las comunas.

No es posible interpretar de manera sencilla los hallazgos de este estudio con respecto a la utilización de servicios del sistema de salud, porque ni la salud ni el uso de servicios - $\mathrm{O}$ su financiamiento- son claramente positivos o negativos. Sin embargo, este estudio identifica un conjunto de desigualdades en la asignación de recursos y en su utilización que requieren mayor investigación para poder entender si están evidenciando inequidades que necesiten ser enfrentadas. Una parte crucial de ese análisis es la comparación de la calidad de los servicios y de resultados específicos de la atención de la salud entre subgrupos de población.

\section{Implicaciones metodológicas}

En relación con el marco teórico basado en las cuatro dimensiones que usamos en nuestro trabajo, consideramos que constituye una herramienta bastante práctica para organizar y presentar datos para el diseño e implementación de políticas de salud. Desde nuestra perspectiva, puede ser útil ampliar dicho marco para incluir una quinta dimensión relacionada con las políticas públicas (por ejemplo, leyes, regulaciones, programas y su implementación tanto en el sector de la salud como más allá del mismo) pues indudablemente afectan a la salud de las personas y de las comunidades. Esta propuesta se refleja en el cuadro 7 , en el que se incluyen algunas preguntas que, desde nuestra perspectiva, pueden orientar futuras investigaciones en el campo de la equidad en salud.

En resumen, los resultados obtenidos sobre la base de información confiable confirman que existen importantes variaciones geográficas en la mortalidad y otros resultados de salud, en ingresos y condiciones ambientales, y en el financiamiento y utilización de los servicios del sistema de salud. Al mismo tiempo, pudimos constatar que la información recolectada de forma regular y que se encuentra disponible

\section{CUADRO 7. Marco teórico para ampliar la investigación sobre equidad en salud}

\begin{tabular}{|c|c|c|}
\hline & & Foco específico \\
\hline Dimensiones de la equidad en salud & Ejemplos de preguntas por dimensión & $\begin{array}{l}\text { Geografía. Ingreso. Género. } \\
\text { Ocupación. Educación. Etnia. } \\
\text { Edad. Vivienda. Ambiente. } \\
\text { Sistema de provisión de la } \\
\text { atención sanitaria. Evaluador. }\end{array}$ \\
\hline I. Estado de salud & $\begin{array}{l}\text { ¿Son similares los patrones de enfermedad, daño y mortalidad entre } \\
\text { subgrupos de población? ¿Varía de manera significativa su tratamiento? }\end{array}$ & \\
\hline II. Determinantes de la salud & $\begin{array}{l}\text { ¿Existen variaciones importantes en términos de factores económicos, } \\
\text { sociales y ambientales? ¿Cómo afecta esto a la salud? }\end{array}$ & \\
\hline $\begin{array}{l}\text { III. Recursos y oferta en el sistema } \\
\text { de salud }\end{array}$ & $\begin{array}{l}\text { ¿Cómo se distribuyen los recursos actuales del sector de la salud? } \\
\text { ¿Satisface la distribución de la oferta las necesidades de la población? }\end{array}$ & Áreas de futura investigación \\
\hline IV. Utilización de los servicios de salud & $\begin{array}{c}\text { ¿Varía de manera significativa el uso de servicios de salud del sector } \\
\text { público debido a factores externos al sector, como los factores } \\
\text { geográficos o los ingresos? }\end{array}$ & \\
\hline V. Legislación y políticas públicas & $\begin{array}{c}\text { ¿Cómo afectan al estado de salud los procesos políticos } \\
\text { estructurados formalmente, las leyes, los presupuestos, las políticas } \\
\text { y su implementación? }\end{array}$ & \\
\hline
\end{tabular}

Fuente: Modificación sobre marco de análisis propuesto por OMS (referencia 19). 
para caracterizar las variables relacionadas con la salud, tiene con cierta frecuencia limitaciones de calidad, sostenibilidad y/o accesibilidad.

Desde la perspectiva de quienes toman decisiones, el desafío es equilibrar los beneficios de la información adicional que se emplea como insumo para mejorar la decisión y los costos en que se incurre para generar dicha in- formación. En el contexto chileno sería infructuoso centrar los mayores esfuerzos en la reorganización de los sistemas de información, toda vez que las pruebas actuales de la existencia de marcadas desigualdades resultan más que suficientes para apoyar la planificación de intervenciones orientadas a mejorar con urgencia la situación de los chilenos más desprotegidos.
Agradecimientos. Agradecemos la colaboración del Ministerio de Salud del Chile, en especial del equipo del Departamento de Epidemiología encabezado por la Dra. Ximena Aguilera. Agradecemos también al Ministerio de Planificación y Coordinación y a la Subsecretaría de Desarrollo Regional y Administrativo del Ministerio del Interior.

\section{REFERENCIAS}

1. Casas JA. Promover la equidad en salud y desarrollo humano en la cooperación técnica en salud en las Américas: la perspectiva de la Organización Panamericana de la Salud. División de Salud y Desarrollo Humano, OPS. Documento presentado en la reunión de Gerentes en la OPS. Washington, D.C., 25-30 de octubre de 1999.

2. Krasnik A. The concept of equity in health services research. Scand J Soc Med 1996;24: 2-7.

3. Oganización Panamericana de la Salud, Comisión Económica para América Latina y el Caribe. Salud, equidad y transformación productiva en América Latina y el Caribe. Washington, D.C.: OPS, CEPAL; 1997 (Cuaderno Técnico No. 46).

4. Farmer P. Social inequalities and emerging infectious diseases. Emerg Infect Dis 1996;2: 259-69.

5. Chile. Ministerio Secretaría General de la Presidencia. Mensaje presidencial, 21 de mayo de 2001. Santiago de Chile: Ministerio Secretaría General de la Presidencia; 2001.

6. Chile. Ministerio de Salud. Políticas de salud y metas ministeriales 2000-2006. Santiago de Chile: Ministerio de Salud; 2000.

7. División de Inversiones y Desarrollo de la Red Asistencial. Sistematización de estudios de red asistencial: visión nacional. DINRED. Santiago de Chile: Ministerio de Salud; 1999.

8. Gilson L. In defence and pursuit of equity. Soc Sci Med 1998;47:1891-1896.

9. Mooney GH. Equity in health care: confronting the confusion. Eff Health Care 1983; 1:179-185

10. Alleyne GA, Casas JA, Castillo-Salgado C. Equality, equity: why bother? Bulletin World Health Organ 2000;78:76-77.
11. Whitehead M. The concepts and principles of equity and health. Copenhagen: WHO Regional Office for Europe (EURO); 1990.

12. Giacomini MK. The which-hunt: assembling health technologies for assessment and rationing. J Health Polit Policy Law 1999;24: 715-758.

13. Mooney G. Judging goodness must come before judging quality-but what is the good of health care? Int J Qual Health Care 2000;12: 389-394.

14. Oganización Panamericana de la Salud: Metodología para la evaluación y el seguimiento de la reforma del sector salud en América Latina y el Caribe. Washington, D.C.: OPS; 1998

15. Dachs N. Measures of inequality in health: what we have, what we need, how can we get there? Documento presentado en la reunión de Gerentes en la OPS, Washington D.C., 25-30 de octubre de 1999.

16. Indicadores/metodologías para medir/establecer equidad en salud. Documento elaborado por el Programa Especial de Análisis de Salud (SHA) para la reunión de Gerentes en la OPS, con base en el Capítulo 1 del Informe Anual del Director de la OPS, 1998: Información para la salud, y presentado en la reunión de Gerentes en la OPS, Washington D.C., 25-30 de octubre de 1999.

17. Anderson NB. Solving the puzzle of socioeconomic status and health: the need for integrated, multilevel, interdisciplinary research. Ann N Y Acad Sci 1999;896:302-312.

18. Krieger N, Rowley DL, Herman AA. Racism, sexism, and social class: implications for studies of health, disease, and well-being. American Journal of Preventive Medicine 1993;9 (supl).

19. World Health Organization. Final report of the meeting on policy-oriented monitoring of equity in health and health care. Geneva: WHO; 1998.

20. González C, Aguilera X, Guerrero A, Child V, Georgi $\mathrm{H}$. Indicadores comunales para el estudio de la desigualdad en salud. El Vigía 2000:3:7-13.

21. Concha M, Aguilera X. Apuntes para una epidemiología de la desigualdad. En: Equidad y eficiencia en la toma de decisiones en salud. Santiago de Chile: Ministerio de Salud, División de Salud de las Personas (DISAP); 1998.

22. Hollstein RD, Vega J, Carvajal Y. Desigualdades sociales y salud: nivel socioeconómico y mortalidad infantil en Chile, 1985-1995. Rev Med Chil 1998;126:333-340.

23. Vega J, Bedregal P, Delgado I, Jadue L. Informe preliminar. Estudio. Equidad y género. Santiago de Chile: Departamento de Salud Pública, Facultad de Medicina, Pontificia Universidad Católica; 2000.

24. Ferrer M, Dachs N. Hoja-resumen sobre desigualdades en salud: Chile. Washington, D.C. Organización Panamericana de la Salud; 2000.

25. Martiny A. The lack of transparency in the health care sector: squandered resources, improper usage, deception - gateways to corruption. [Sitio en Internet] Transparency International. Disponible en: http://www. transparency.org/documents/work-papers/ health care.html. Acceso el 26 abril 2002.

26. Yen IH, Syme SL. The social environment and health: a discussion of the epidemiologic literature. Ann Rev Public Health 1999;20:287-308.

27. Larrañaga O, Infante A. Descentralización de la atención primaria de salud en Chile. Santiago de Chile: Departamento de Economía, Facultad de Ciencias Económicas y Admnistrativas, Universidad de Chile; 2000. 
ABSTRACT Objectives. To estimate the magnitude of geographical health inequalities in Chile through key indicators based on data and information that are routinely collected and easily obtained, and to characterize the current situation with respect to the availability, quality, and access to information on health equity that official sources routinely collect. Methods. A conceptual framework proposed by the World Health Organization was used to study health equity in terms of four dimensions: 1) state of health, 2) health determinants, 3) resources for and the supply of health system services, and 4) utilization of health system services. For each of these four dimensions, indicators were selected for which there was available information. The information was aggregated according to geographical and administrative units in the country: communes (342 in Chile), sanitary districts called "Health Services" (28), and regions (13). The aggregated information was analyzed using univariate analysis (distribution characteristics), bivariate analysis (correlations and frequency tables), and tabulation of values for selected indicators for the communes.

Results. With respect to the first dimension, state of health, we found an inverse relationship between mortality and average family income in the communes $(r=-0.24 ; P<$ $0.001 ; n=191$ communes). With health determinants, there were important differences among the communes with regard to average household income, years of schooling, literacy, quality of housing, drinking water supply, and the wastewater disposal system. In terms of resources for and the supply of health system services, the municipal governments of the communes with higher average household incomes tended to contribute more funds per beneficiary $(r=0.19 ; P=0.013)$. The financial contributions from the national government were targeted well, but they only partially compensated for the morelimited resources available in poorer communes. With respect to the utilization of health care services per beneficiary in the different sanitary districts, we found some large differences. In terms of the ratio between the highest rate of utilization in any of the districts and the lowest rate in any other district, the ratio for primary-care visits per beneficiary was 2.8 , the ratio for emergency-care visits was 3.9, and the ratio for hospitalizations was 2.0.

Conclusions. There are important geographical differences in Chile with respect to mortality and other health outcomes, income and environmental conditions, and the financing and utilization of health care services. The information that is collected regularly and is available to characterize the health-related variables frequently has limitations in terms of quality, sustainability, and access. In Chile it would be pointless to focus the greatest efforts on reorganizing the information systems. The existing indicators showing marked inequalities are adequate to support the planning of interventions aimed at making urgently needed improvements in the situation of the worst-off Chileans.

\title{
International Women and Health Meeting
}

\author{
Dates: $\quad$ 12-16 August 2002 \\ Location: York University, Toronto, Ontario, Canada
}

The 9th International Women and Health Meeting (IWHM) will focus on action and the development of strategies, solutions, and approaches to the promotion of women's health. The IWHMs are based on the recognition of equality as a condition of health and the recognition of the principle of distributive justice for women of the North and of the South. The 9th IWHM will focus on three themes: sexual and reproductive rights, State and family violence against women, and environmental health.

The 2002 meeting will include: plenary sessions, where links will be made among two or three of the themes of the meeting; workshops to construct strategic action plans applicable at the local, regional, and international levels; workshops to identify the sectors where recommendations need to be made with respect to current policy, or where new policies are needed; workshops that will enable women to upgrade their skills in using the Internet; workshops that will help identify action priorities for the future; and an exhibition fair to share information on the work done by groups all around the world.

The meeting is intended for women who are involved in the women's health movement, who are activists within their countries, who represent community groups offering health and social services, or who have adopted a feminist approach and want to share action strategies for advancing women's rights to health.

The registration fee for the entire conference is US\$ 250 . Participants can also choose to attend individual days, for US\$50 per day.

Information (in English, French, and Spanish): 9th IWHM Secretariat

Riverdale Immigrant Women's Centre (RIWC)

1326 Gerrard Street East, Toronto, Ontario M4L 1Z1, Canada

Telephone: (416) 465-6021 Fax: (416) 461-4662

E-mail: info@iwhm-rifs.org

Internet: http://www.iwhm-rifs.org/index.htm 